\title{
5-Aminolevulinic acid-assisted resection of pediatric dysembryoplastic neuroepithelial tumor: illustrative case
}

\author{
Luke H. Beauchamp, ${ }^{1}$ Marian Michael Bercu, MD, MSc, ${ }^{2}$ and Anthony M. Avellino, MD, MBA ${ }^{1,2}$ \\ ${ }^{1}$ College of Human Medicine, Michigan State University, East Lansing, Michigan; and ${ }^{2}$ Pediatric Neurosurgery, Helen DeVos Children's Hospital, Spectrum Health, \\ Grand Rapids, Michigan
}

\begin{abstract}
BACKGROUND 5-Aminolevulinic acid (5-ALA) is approved as an adjunct for the resection of high-grade gliomas and is associated with improved outcomes. Dysembryoplastic neuroepithelial tumors (DNETs) are benign glioneural tumors occurring primarily in pediatric patients and often manifesting with seizure disorder. The goal of the surgical intervention is to obtain gross-total resection, which is associated, in the majority of cases, with seizure freedom.
\end{abstract}

OBSERVATIONS The authors present the first case report of a pediatric patient who underwent gross-total resection of a 5-ALA-positive DNET with no evidence of recurrent seizures (Engel class I).

LESSONS Fluorescence-guided surgery using 5-ALA facilitated gross-total resection of the mass.

https://thejns.org/doi/abs/10.3171/CASE20153

KEYWORDS 5-aminolevulinic acid; 5-ALA; DNET; Gleolan; dysembryoplastic neuroepithelial tumor; fluorescence

Fluorescence-guided resection of gliomas using 5-aminolevulinic acid (5-ALA) has proved to be effective in extending the positive effects of resection. ${ }^{1}$ We present the unique case of a pediatric patient with a grade 1 dysembryoplastic neuroepithelial tumor (DNET) in the left occipital lobe that showed positive 5-ALA fluorescence. To our knowledge, this is the first reported case of a 5-ALA-positive DNET. This report contributes to the literature suggesting the usefulness of 5-ALA fluorescence to guide resections for low-grade brain tumors.

\section{Illustrative Case}

DNETs are a rare, benign glioneural tumor occurring primarily in pediatric patients and often manifesting in seizure disorder. The goal in DNET surgery is complete removal because residual tumor correlates with the return of seizures. ${ }^{2}$

Fluorescence-guided surgery (FGS) using 5-ALA enables more extensive resection of malignant gliomas in adults, associated with longer progression-free survival. ${ }^{3}$ 5-ALA works through the porphyrin synthesis pathway, resulting in an accumulation of the fluorescent compound protoporphyrin IX, with high sensitivity, specificity, and positive predictive value for tumor identification. ${ }^{1}$

Similar reliability and utility have not been demonstrated for 5ALA in the resection of pediatric gliomas. ${ }^{4}$ The consensus appears to be that 5-ALA works with varying utility for such lesions;, 5 however, those conclusions are based on very limited published data. In this report, we describe a pediatric DNET that was 5-ALA positive, which helped guide our gross-total resection.

\section{History and Examination}

The patient was a 10-year-old male who presented with medically intractable complex partial seizures and visual deficits. The results of his physical and neurological examinations were grossly within normal limits, with a suggestive right homonymous hemianopsia visual field defect per Humphrey visual fields. The patient's electroencephalogram was abnormal, suggestive of a seizure disorder. Brain magnetic resonance imaging (MRI) revealed a large, solid,

ABBREVIATIONS 5-ALA $=$ 5-aminolevulinic acid; DNET = dysembryoplastic neuroepithelial tumor; FGS $=$ fluorescence-guided surgery; IDH $=$ isocitrate dehydrogenase; $\mathrm{MRI}=$ magnetic resonance imaging.

INCLUDE WHEN CITING Published online July 26, 2021; DOI: 10.3171/CASE20153.

SUBMITTED December 21 2020. ACCEPTED February 152021.

(c) 2021 The authors, CC BY-NC-ND 4.0 (http://creativecommons.org/licenses/by-nc-nd/4.0/). 
and cystic mass in the left occipital lobe measuring $6.0 \times 4.4 \times$ $4.1 \mathrm{~cm}$. The mass exhibited moderate gradient susceptibility and cortically based nodular enhancement that involved the subcortical white matter (Fig. 1A). Vasogenic edema and restricted diffusion were negligible. This constellation of imaging findings, age, and presentation were thought to be consistent with a slower-growing lowgrade tumor. The differential diagnosis in a patient of this age favored oligodendroglioma, pleomorphic xanthoastrocytoma, DNET, or ganglioglioma; however, higher-grade glioma could not be ruled out.

\section{Operation}

The patient received oral administration of 5-ALA before FGS $(20 \mathrm{mg} / \mathrm{kg}$ approximately 120 minutes before anesthesia). A gross-total resection was completed via a posterior left craniotomy (Fig. 1B). The tumor demonstrated positive fluorescence,
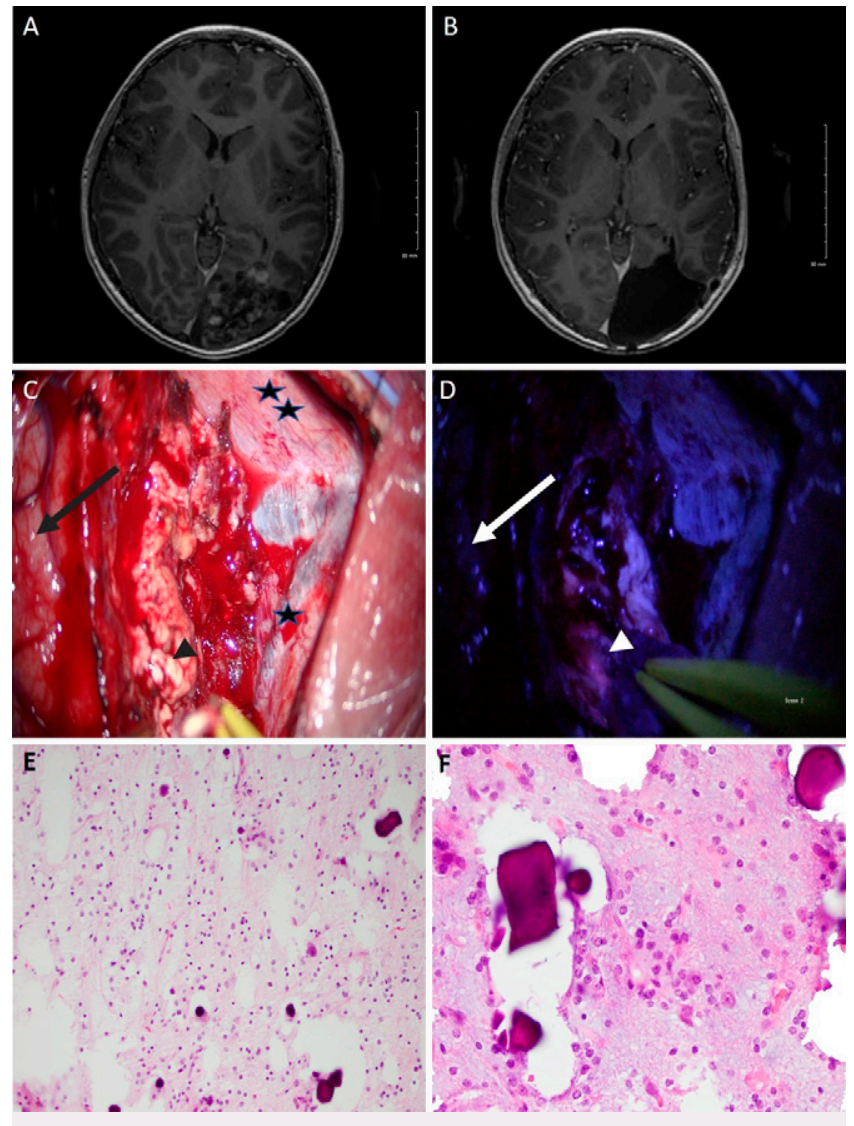

FIG. 1. A: Preoperative axial gadolinium (Gd)-enhanced T1-weighted MRI of a nonhomogeneously enhancing mass encompassing most of the left occipital lobe. B: Postoperative axial Gd-enhanced T1-weighted MRI demonstrating gross-total resection of the mass. C: Intraoperative view of the mass. Arrow indicates normal brain tissue; arrowhead indicates abnormal mass; single star indicates tentorium cerebelli; double star indicates the falx. D: The mass showing typical charcoal-red fluorescence under a 440-nm ultraviolet light source of the intraoperative microscope. Arrow indicates normal brain tissue; arrowhead indicates the mass. E and F: Photomicrographs of hematoxylin and eosin staining demonstrating the typical appearance of a DNET, including the presence of glioneural columns with small, bland cells lining vascular elements and neuronal processes. Original magnifications $\times 40(E)$ and $\times 200(F)$ Original magnifications $\times 40(E)$ and $\times 200(F)$. which guided our gross-total resection (Fig. 1D). The positive fluorescence extended notably beyond the borders of the macroscopically abnormal tissue, largely correlating with the neuronavigation-suggested borders, which can be unreliable in the course of resection of large and very large masses.

The histopathological finding was DNET, World Health Organization grade 1 isocitrate dehydrogenase (IDH) R132 wild type. Immunohistochemistry revealed glial fibrillary acidic protein and $\mathbf{S 1 0 0}$ positivity, epithelial membrane antigen negativity, IDH1 negativity, Ki67 proliferation index $2 \%-5 \%$, and p53 $<1 \%$ positive nuclear staining. Additional findings were extensive calcifications, fine vasculature, and small round glial cells with rare floating nuclei (Fig. 1E and F).

At the 6-month follow-up, the patient remained seizure free and was currently off all antiepileptic medications. The patient experiences persistent right homonymous hemianopsia, and his brain MRI revealed a gross-total resection (Fig. 1B). He will continue to be seen in follow-up with serial brain MRI.

\section{Discussion Observations}

In this study, we present a pediatric patient with the first instance of a 5-ALA-positive DNET. Fluorescence is considered to be associated with the hallmarks of high tumor grade, including higher cell proliferative activity, neovascularity, and blood-brain barrier permeability.7,8 Macroscopic fluorescence has been historically associated with a low rate of positivity in low-grade pediatric tumors, with the value of FGS in children being primarily for the resection of highgrade gliomas. 5

High-grade tumors exhibit lower amounts of ferrochelatase. Decreased levels of ferrochelatase lead to the intracellular accumulation of protoporphyrin IX.9 Low-grade pediatric tumors have demonstrated limited fluorescence using standard microscopic visualization. Notably, intracellular protoporphyrin IX accumulation can be detected in many low-grade tumors, even when visible fluorescence is absent, using the more sensitive confocal microscopy via a handheld fiberoptic probe.6,10 However, even with these more sensitive techniques, protoporphyrin IX accumulation has not been reported in DNETs.

The ability to identify fluorescence in a DNET is significant, given that FGS may be helpful in selected cases to obtain a safe complete resection. Although DNETs are nonmalignant, residual tumor is associated with recurrent seizures and the need for a repeat surgery. ${ }^{11}$ In addition, a potential explanation for fluorescence would be the presence of anaplastic tumor cells. ${ }^{12}$ However, no anaplastic foci were seen in the histopathological evaluation. We contemplated more aggressive postoperative therapy, given the unexpected presence of 5-ALA, but we relied on histopathology. We did not want to subject the patient to unnecessary morbidity, given the benign nature of DNETs, and decided to continue with serial brain MRI.

The limitations of the presented data are related to the nature of a case report and possible inaccurate pathological misdiagnosis, including sampling error. In addition, autofluorescence of brain tissue has been reported as a cause of false-positive fluorescence but is exceedingly rare and would be more focal than was demonstrated in our patient. ${ }^{13}$ Further data from similar cases may be helpful in determining the prevalence of 5-ALA tumor positivity in the pediatric population. 


\section{Lessons}

This is the first reported case of 5-ALA-positive fluorescence in a DNET. A single report does not allow us to comment on the prognostic significance of 5-ALA positivity. Recognizing the presence of fluorescence may suggest the need for careful reexamination of histopathology and closer follow-up. However, it should now be recognized that 5-ALA positivity does not exclude the diagnosis of DNET, and, equally important, 5-ALA should be considered as a surgical tool to improve the likelihood of a gross-total resection, as in this case.

\section{References}

1. Stummer W, Pichlmeier U, Meinel T, et al. Fluorescence-guided surgery with 5-aminolevulinic acid for resection of malignant glioma: a randomised controlled multicentre phase III trial. Lancet Oncol. 2006;7(5):392-401.

2. Englot DJ, Berger MS, Barbaro NM, Chang EF. Factors associated with seizure freedom in the surgical resection of glioneuronal tumors. Epilepsia. 2012;53(1):51-57.

3. Zhang C, Boop FA, Ruge J. The use of 5-aminolevulinic acid in resection of pediatric brain tumors: a critical review. J Neurooncol. 2019;141(3):567-573.

4. Schwake M, Schipmann S, Müther M, et al. 5-ALA fluorescenceguided surgery in pediatric brain tumors-a systematic review. Acta Neurochir (Wien). 2019;161(6):1099-1108.

5. Roth J, Constantini S. 5ALA in pediatric brain tumors is not routinely beneficial. Childs Nerv Syst. 2017;33(5):787-792.

6. Stummer W, Tonn JC, Goetz C, et al. 5-Aminolevulinic acid-derived tumor fluorescence: the diagnostic accuracy of visible fluorescence qualities as corroborated by spectrometry and histology and postoperative imaging. Neurosurgery. 2014;74(3):310-320.

7. Ennis SR, Novotny A, Xiang J, et al. Transport of 5-aminolevulinic acid between blood and brain. Brain Res. 2003;959(2):226-234.
8. Stummer W, Reulen HJ, Novotny A, et al. Fluorescence-guided resections of malignant gliomas-an overview. Acta Neurochir Suppl (Wien). 2003;88:9-12.

9. Zhao SG, Chen XF, Wang LG, et al. Increased expression of ABCB6 enhances protoporphyrin IX accumulation and photodynamic effect in human glioma. Ann Surg Oncol. 2013;20(13):4379-4388.

10. Valdés PA, Leblond $F$, Kim A, et al. Quantitative fluorescence in intracranial tumor: implications for ALA-induced PpIX as an intraoperative biomarker. J Neurosurg. 2011;115(1):11-17.

11. Chassoux F, Daumas-Duport C. Dysembryoplastic neuroepithelial tumors: where are we now? Epilepsia. 2013;54(suppl 9):129-134.

12. Widhalm G. Intra-operative visualization of brain tumors with 5-aminolevulinic acid-induced fluorescence. Clin Neuropathol. 2014;33(4):260-278.

13. Panciani PP, Fontanella M, Schatlo B, et al. Fluorescence and image guided resection in high grade glioma. Clin Neurol Neurosurg. 2012;114(1):37-41.

\section{Disclosures}

The authors report no conflict of interest concerning the materials or methods used in this study or the findings specified in this paper.

\section{Author Contributions}

Conception and design: all authors. Acquisition of data: Bercu, Avellino. Analysis and interpretation of data: all authors. Drafting the article: all authors. Critically revising the article: all authors. Reviewed submitted version of manuscript: all authors. Approved the final version of the manuscript on behalf of all authors: Bercu. Administrative/technical/ material support: Bercu. Study supervision: Bercu.

\section{Correspondence}

Marian Michael Bercu: Spectrum Health, Grand Rapids, MI. marian. bercu@spectrumhealth.org. 